\title{
Survey on Nano Technology
}

\author{
Adel Alfoudery ${ }^{* 1}$, Abdulrahman Alkandari², Samer Moein ${ }^{3}$ \\ ${ }^{1,2}$ Computer Department, College of Basic Education, (PAAET), Kuwait \\ ${ }^{3}$ Electrical and Computer Engineering, University of Victoria, Canada
}

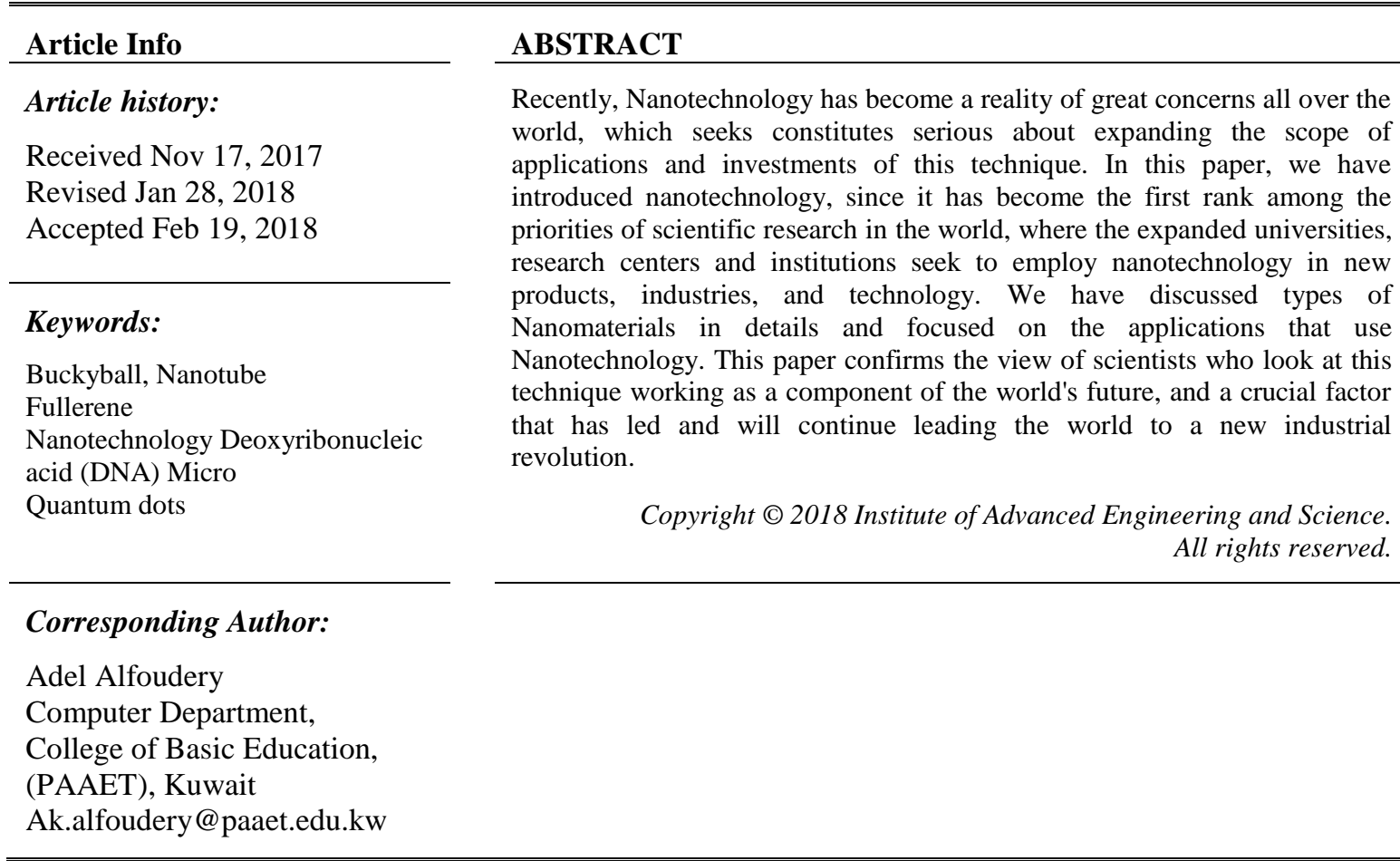

\section{INTRODUCTION}

Microparticles in the Nano-technology refer to handling materials at the atomic and molecular scale. Nanotechnology results of many complicated research studies that deal with nanotechnology between 0.1 to 100 nanometer (a nanometer is a part of the thousand-millionths of a meter) standards of any agreement, with the nuclear concentration ranging between five thousand atoms. It is smaller than the tiniest bacterium [1]. "This selection offsets the broadening nature of the applied materials. Nanotechnology includes a treat with the phenomena of any buildings or on the level of small Nano. Such nanoscale phenomena are able to comprise a quantitative constraint that leads to the electromagnetic and an optical new material, that has been evaluated between particle size and the size of the solid visual phenomena. Phenomena nanoparticles moreover comprise the impact of the Gibbs-Thomson [2] - a low melting point of a substance when it becomes measurable Nano, either building. The most important are Nano carbon tubes.

Nanotechnology has a strain which comes from the probability to control the atoms formed after segmentation including the materials. Consequently, it needs to be very specific devices of hand size, standards, and methods of seeing the molecules under checkup. After reaching the level of the atom, the strain of reaching a precise measurement is an additional struggle added to this new emerging science. Furthermore, there is still disagreement and worries about the effect of nanotechnology [3].

The theory of nanotechnology relies on the basis that particle size of fewer than one hundred nanometers provides a material containing the structure and properties of different behaviors. However, these particles illustrate new physical and chemical theories; causing innovative behavior relies on particle sizing. It has been detected, for instance, the electronic structure, interactive, connectivity, the degree of fusion and mechanical properties of the material are all altered when smallest particle size for a critical scale value. The closer the volume of material of atomic dimensions whenever subject material of laws of quantum mechanics rather than traditional physics laws. The behavior of material depends on the size of engineering properties of 
the control. Consequently, finally, the researchers determined that this theory is a worthy technique effect on an extensive variety of technical ranges, which includes the production of light and strong material, reduced drug delivery time, nanoscale to the human circulatory system, increasing the size of the absorption of magnetic tapes... etc. In general, nanotechnology deals with several combinations of dimensions of the rank of nanometer materials [4].

Nevertheless, the statement that nanotechnology is quite innovative, the existence of devices work with this theory and structures with nanoscale dimensions is not innovative. It is recognized that biological systems in vivo production some small devices which up to the limits of the nanoscale. A living cell is the main case of the technique ordinary nanotechnology, which it is a cell origin of a big number of biological machines. Nano size is the synthesis of proteins inside the form of lines joined the Nano size named (liposome), then it is designed by the last Nano named (Golgi apparatus), but the Enzymes which are Nanomachine itself is the separation of the particles collected by the need or the cell. Thus, it can be for machines manufactured nanomaterials that work together with them and achieve the anticipated goal, for example, the analysis of the cell contents, drug delivery to or destruction when they become harmless [5].

It is also not known to the launching of the human usage of the material with nanoscale size. However, it is recognized that one of the holdings of glass, a Roman King Cup in the Museum of England comprises particles Gold and Silver Nano, which the cup color changed from green to dark red when the light source is located. In addition, from the time when the eighteenth and nineteenth century's photography technique depends on producing a film is made of silver nanoparticles are sensitive to light [5].

\section{HISTORY OF NEW TECHNOLOGY}

Nanotechnology is a relatively new emerging technique in the next ten years. Actually, it became a phenomenon. In addition, development has continued until today, where more than 2000 of only 4004 transistors in processors for the year 1971 have been increased to more than 700 million transistors in Intel Core 2. There was, of course, a similar reduction in the size of individual electronic items, and go from millimeters to hundreds of nanometers in electrical circuits. At the beginning of the new century, three powerful technologies have met its scope on a joint-Nano-scale, which is a revolution in the world of electronics and biology [6].

At the same time, chemistry, physics, organic chemistry and atomic elements and genetic engineering has taken other paths in the same time period and has become possible to guide the vehicles either in test tubes or living organisms themselves [7].

With the beginning of the three powerful current century technologies amounted to public standards standards of nanotechnology - with promises in both global electrons and neighborhoods has been developed. These new fields are vital parts precision. The Technology is carrying several possibilities of research in the field of molecular biology [3].

\section{TYPES OF NANOMATERIALS}

\subsection{Quantum Dots}

Nano semiconductor has three-dimensional ranges dimensions between two to ten $\mathrm{nm}$, and the point of quantitative restriction of conduction electrons bar and holes parity or position bar (It is a linked pair of conduction electrons and holes parity). The show point's quantity spectrum is energetic gagged spotty, and the corresponding wave functions are stationed inside the point quantity. When the diameter of the point amount is equal to $10 \mathrm{~nm}$, it can collocate 3 million a number of points next to each other, with a length equal to the width of human finger thumb.

\subsection{Fullerene}

Installation of Nano strange last carbon is a molecule composed. Since the discovery of Fullerene manufacturing in 1990, which brings in commercial quantities, it is possible to get the number of different molecules of carbon atoms, such as $\mathrm{C} 36$ and $\mathrm{C} 48$ and C70, but scientists have shown special interest molecule C60. Installation of Fullerene proportion has been called to the inventor and architect R. Buckminster Fuller (R. Buckminster Fuller). So, he grew up a new branch of chemistry called Fullerene, knew where more than 9,000 composite Fullerene since 1997, and different applications for each of these vehicles appeared, including vehicles and $\mathrm{K} 3 \mathrm{C} 60 \mathrm{RbCs} 2 \mathrm{C} 60$ and $\mathrm{C} 60-\mathrm{CHBr} 3$ that have shown superior connectivity (superconductivity). Other forms of Fullerene cone and tubular has also been discovered in addition to football [6]. 


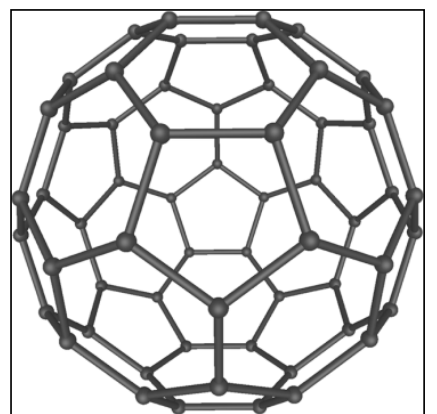

Figure 1. Fullerene Carbon Nanotubes

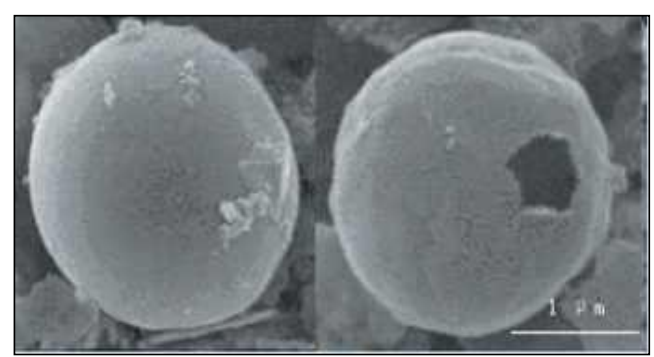

Figure 2. Illustrative Nano Ball

\subsection{Nanoballs}

Nano-carbon balls are the most important type that belongs to the fullerene category of material C60 but differ slightly since it formulators multiple crusts. It is also an empty place, whereas the surface has no gaps, as is the situation in multiple cover nanotubes (Figure 1). The diameter of the balls up to $500 \mathrm{~nm}$ nanoparticles or more [5]. Figure 2 shows an illustrative image for Nano ball.

\subsection{Nanoparticles}

Although the modern use of nanoparticles, these particles were present in processes or natural materials since ancient times. For example, some of the beautiful colors of glass windows and rusty, because of the occurrence of small clusters of metallic oxides in the glass where the sizes are near enough the wavelength of light. Thus, the different sizes of particles distract the different wavelengths of light, causing the emergence of different glass colors [8].

Nanoparticles (as shown in Figure 3) be able to be described as a collecting atomic or molecular microscopic which can be ranged in size from a few atoms to a million atoms, associated almost spherical radius of less than $100 \mathrm{~nm}$. It just could manufacture nanoparticles of metals and insulators, semiconductors and hybrid combinations (such as nanoparticles coated), as well as models of Nanoparticles with a seminature - a solid manufacturing liposomes. Other images of nanoparticles are the points quantity and semiconducting nanocrystals. The nanoscale copper particles that are up to less than $50 \mathrm{~nm}$ with high hardness and non-ways unlike what happens to ordinary copper material where it can be bent, and pulled easily [8].

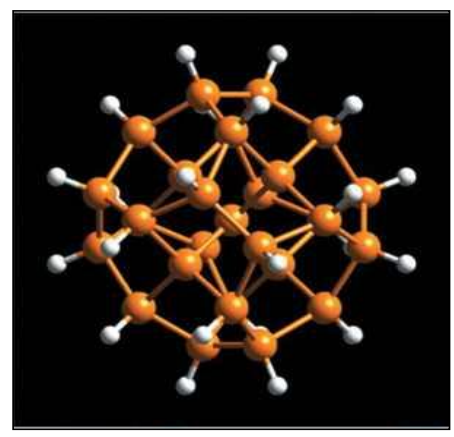

Figure 3. Nanoparticle

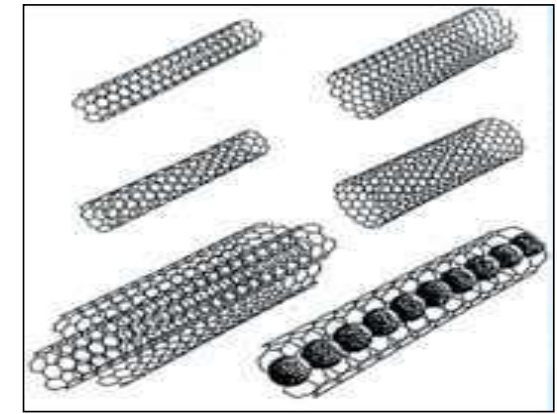

Figure 4. Models for carbon nanotubes

\subsection{Nanotubes}

Nanotubes are nanoparticles of carbon discovered in 1991. Nanotubes have more importance due to their characteristics and use extensive applications in industrial, scientific, medical and bio-electronic devices. Nanotubes are able to be defined as a carbon nanotube where a graphite segment is folded around the axis of a cylindrical shape, where atoms are linked with each end of the slide to close the tube. The tube ends might be in the form of a hemisphere. It might also be separate atoms tube wall, which is termed in this case nanotubes and single wall nanotube (SWNT), or binary or more which is termed multi-wall tubes 
(MWNT), with tube diameter, sorts from less than one nanometer to 100 nanometers, but it has a length of up to 100 micrometers to form Nanowire [2] as shown in Figure 4.

\subsection{Nanofibers}

Fiber nanoparticles are those made of atoms polymers. The surface area is relative to the size in the case of fiber. Nanoparticles and Nano-tubes have the number of large surface atoms compared to the total number, and this makes such fibers distinctive mechanical properties. For example, hardness and tensile strength, with no competitor for the usage as filters in the refinement of gases or liquids. In biomedicine organ transplantation, such as arthritis and transport of drugs in the body and military use such as reducing air resistance, especially after the development of methods of preparation. Figure 5 shows fiber nanoparticles.

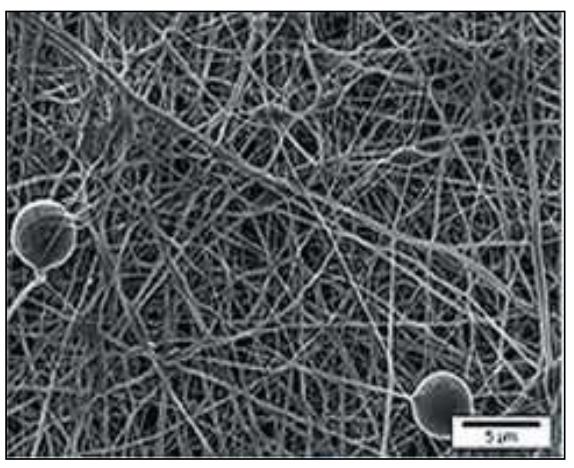

Figure 5. Fiber Nanoparticles [3]

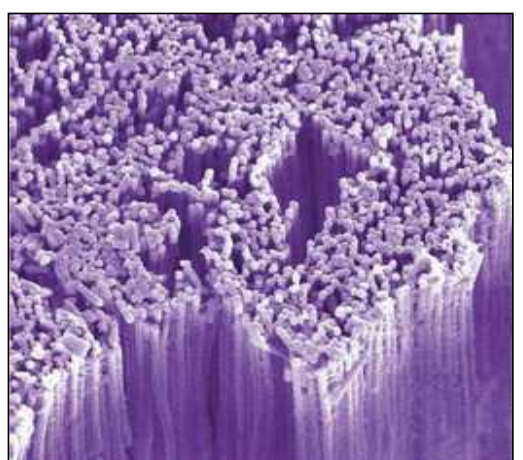

Figure 6. Nanoscale Wires

\subsection{Nanowires}

In Nanowires, the wire diameter may be less than one nanometer. As a result, the electrons are limited measured toward both sides which make it occupy exact levels of energy, which vary from those extensive levels found in volumetric of the article. Because of its submission of quantitative restriction built on quantum mechanics, it will have electrical conductivity which take exact values almost equal to complications amount to 12.9 kilo-ohm 1, which does not exist in nature but can be urbanized in the laboratory, where the metallic ones (nickel, silver, platinum), semiconductor and separation (silicates, titanium oxide), including organic molecular wires (DNA) can be used. This can be done, to tie accurate electronic components inside a small circle or the work of bilateral links, as well as establishing the logical electronic circuit may be utilized for the manufacture of the digital computer in the future. The expected electronic uses are too many, which will achieve molecular Nano-bio-sensors [9]. Figure 6 shows Nanoscale wires scanning with the electron microscope.

\subsection{Nan Composites}

Nan composites is a material addition to nanoparticles. Through the production of those materials, and accordingly, article nanoparticles indicate an important enhancement in their properties. For instance, adding carbon nanotubes to change the electrical conductivity and thermal properties of the material. Other types of nanoparticles were added to enhance the optical properties and electrical insulation properties, as well as mechanical properties such as hardness and strength. The volumetric percentage of nanoparticles started to be very low (in the range of $0.5 \%$ to $5 \%$ ) due to the ratio of surface area to the size of nanoparticles be high [6].

Research is currently underway for a new Nano-compounds with the characteristics and features differ from the original vehicles. It is now known that nanoparticles compounds are polymeric compounds nanoparticles.

\section{APPLICATIONS OF NANOTECHNOLOGY}

There are many of Nanotechnology applications around us in our life. In this part, we discuss some of them.

\subsection{Medical Applications}

This technique can be used for imaging the body's cells without difficulties to take a normal picture, also, is able to control these cells in other ways. 
Several types of nanoparticles are used in medical uses which can be as bearers of the drug or tools for imaging inside the body. Presently, they are used for different types of particles liposomes manufactured nanomaterial's as systems connected to the anti-cancer vaccines medicines, as gold nanoparticles used in home testing devices for the detection of pregnancy [10].

Nanowires are used as sensors vital Nano so high size nanoscale to its sensitivity, where the coating of these nanowires antibodies manufactured so that they only vital molecules stick (DNA), or proteins, or other biological inside the body particles, and no other molecules, and when linked these proteins or other nanowires coated the connection will change, and so it can use this vital nano scale sensor to detect a large number of diseases in their early stages, and that the introduction of large numbers of nanowires inside the body is painted with different antibodies, representing different sensors [11].

Researchers attempt to use the experimental Silicon Nano-capsules to stop the body's immune system to recognize foreign cells, where these capsules are blocking antibodies produced by the body's immune system, while the launch of a sufficient amount of insulin by mobile capsules nanoparticles in the blood [11].

The surgical instruments are now an objective for the development and optimization of nanotechnology. It is predictable in the future to provide positive solutions to correct the loss caused in the audiovisual and sensory organs in the human body and the cultivation of minutes nanoscale devices within the body. For instance, researchers are now working on the cultivation of Nanomembrane in the retina of the blind to improve the vision for the patients.

Bio-Nano-generators, as nanoscale electrochemical devices, produce electrical ability of blood glucose in the body and then utilizes this ability to run different nanoscale devices planted within the human body, for instance, pacemakers devices or robots sugar nanoparticles injection.

A promising medical application of nanotechnology is using a polymer Nano-fibres to make prosthetic blood vessels. In recent times, growing prosthesis devices made of nanoscale protein fibers are used in the central nervous system of a human body.Furthermore, the polymer Nano-fibres are used in the treatment of burns, wounds, and intervention in cosmetics industry [7].

When injected cadmium selenide nanoparticles (points quantity) within the body, they accumulate within cancer cells selectively in the case of exposure to the target region ultraviolet light, particles light up, assisting in defining the site of malignant cells and removing them carefully. Figure 7 shows the schematic illustration to explain how to use nanoparticles or other cancer drugs to treat cancer.

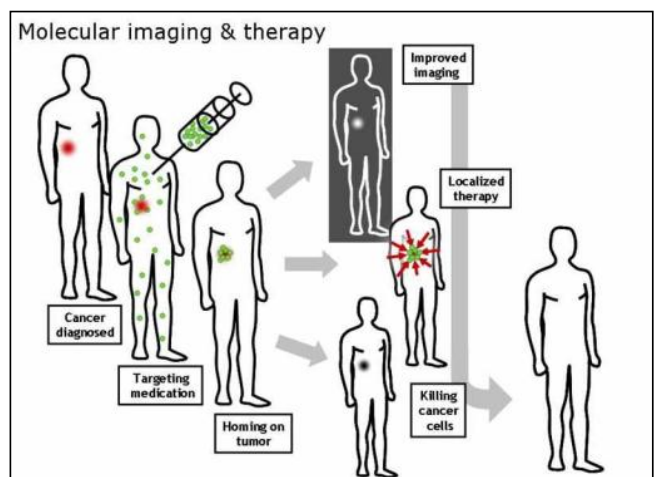

Figure 1. Use of Nanoparticles or Cancer Drugs to Treat Cancer

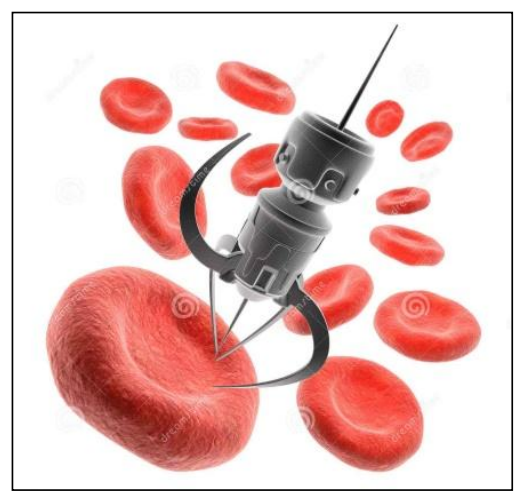

Figure 2. Nanotechnology Used to Treat Blood

Scientists are thinking of how to manufacture mechanical and electrical properties to solve a substitute for red blood cells as shown in Figure 8. Nanotechnology offers an alternative to cut human parts efficiently and be close to original, where the target now is to replace some members that achieve the functions of mobility, such as bone and muscle and joint members of Nanoscale.

The used nanoparticles are made of rare earth elements to take away the phosphate in the blood of patients who have an excess of phosphate in the blood.

Nanotechnology acting an important part of improving the living tissue engineering and cell therapy, which consists of the usage of living cells or natural compounds manufactured within the human living [5].

The benefits of nanoparticles, for example, provide a strong evidence of an increase in the surface area to volume ratio. It then becomes the optical properties such as a fluorescent function of the particle diameter. When joined with mass in the material, the nanoparticles affect the mechanical properties of materials, 
together with the hardness or softness. For example, it can consolidate the traditional polymers through the usage of nanoparticles in the new substances that could be utilized as replacements lightweight metals. As a result, it can be expected to increase the social interest of nanoparticles. It will allow those materials supported Nano to reduce the weight associated with an increase in the stability and improvement of the job. This, in addition to that nanotechnology process, represents an essential growing ability to accurate the material handling. Thereby, providing a range of possibilities, and that was not for others previously imaginable - so it is not surprising that a few areas of human technology excluded from the benefits arising from the use of and the application of nanotechnology.

\subsection{Communications and Computer Applications}

Now nanoscale optical fibers to be able to send information and calls directly with no need to transform light to electricity, consequently increasing the transfer speed to approximately 100 times. The industry is able to nanoscale lasers, making small communications devices. Nanoparticles such as fiber optic convoys in liquid crystals in optical communications are also utilized. The carbon nanotubes are used in transistors impact areas and convoys in computers [13], leading to a significant improvement of the regular material.

The objective of computers' makers at present is to raise the number of convoys in the chipset. The carbon nanotubes with a diameter $2 \mathrm{~nm}$ have a very low resistance, and thus bring a large current, so it can be utilized, such as internal connections in the caravan substitute for ordinary copper wire, and as an effect of the high conductivity of the pipes carbon nanoparticles, they can act as a thermal bath to waste heat away from the chip. Computers are able to be the future manufacturing of carbon tubes.

\subsection{General Application of Nanotechnology}

Recently, certain types of dresses manufacturers used installed nanoparticles such as silicon particles, where these clothes are characterized as resistant to odor and dyes.

Some crystalline nanomaterials gelatinous also used insulation coating the surfaces of buildings and offices for relief from the heat. In addition, some nanomaterials such as tungsten carbide and titanium carbide are characterized by high hardness compared to normal materials, so they are used in producing some cutting and drilling tools.

The television screens analysis accuracy is heavily dependent on the material of phosphorus in the form of a very young age dots, called pixels. When using phosphorus nanomaterials such as selenide zinc, zinc sulfide, and cadmium sulfide in the manufacture of display screens, the analysis and serenity accuracy will frequently be improved, in addition to reducing the cost of production. Samsung Korean company has been able to produce flat-screen display using electronic emission output of carbon nanotubes. Another Japanese company also succeeded in producing pipe lamps cycle depending on the electron emission from carbon nanotubes and features of these lamps to be shinier, efficient and live longer [12].

Some crystalline nanomaterials show abnormal magnetic properties, and these properties are used in many applications, such as submarines, automotive switches, electrical ground power generators, ships engines, and magnetic resonance analysis devices.

Nanotechnology is widely used for the use of nanoparticles and carbon nanotubes in desalination and water purification, making easy access to drinking water in developing countries. In addition, silver nanoparticles in air filters are used to get rid of smells spam and kill germs. It has been found that the use of such filters nanoparticles leads to killing $99 \%$ of the flu viruses lingering in the air. Other applications of the carbon nanotube are used in the manufacture of air filters, to get rid of the carbon dioxide emissions from power plants gas [11].

Many researchers are currently underway to the possibility of using nanoparticles to identify targets in military areas [14], or in vehicles for security purposes, as well as the manufacture of computers with high capabilities for"encryption and decryption operations, and provide military cloths made from nanomaterials appropriate solution for the prevention of bad weather and chemical, nuclear and biological hazards that may face the soldiers in wars.

The polymer grafted tubes of carbon nanoparticles are not implemented in electromagnetic energy because it is a good conductor of electricity. Therefore, it is used as proof of electromagnetic radiation and utilized militarily to protect electronic devices and communications equipment on the battlefield of radiation that comes from bombs and others.

The coating inside refrigerators is a type of silver nanoparticles, which helps to kill germs very accurate and thus lead to maintain the food fresh for a long time, as well as washing machines that use coated particles in order to help in fully laundry.

Intervention vehicles use nanoparticles plastic in the automotive industry, in some parts of the resistance to shocks in the car and its strong and light resistant to rust. 
Since the wire and nanotubes change of electrical resistance when exposed to gases and alkaline halogens, have been chemical sensors manufactured for use in some areas such as detecting leaks of gases, medical surveillance, monitoring of environmental and industrial hazards. Currently, companies are seeking to use Nano-sensors with a small size and high sensitivity in electrical power consumption.

Nanoparticles can be used in battery carbon tubes [15], where it lithium is used inside the pipe storage. It is also used in fuel cells for hydrogen storage. In addition, the transistor made from semiconducting carbon nanotube has proved its effectiveness, such as a sensitive detector for a number of different gases.

There are other areas of Nanotechnology, including organic light-emitting diodes [16], which are used in displays, film photovoltaics, which convert light into electricity, windows coated scratch-resistant and cleans itself by UV, manufacturing smart clothes regulate temperature and measure the pulse and respiration manufacturing, tinted glass manufacturing where heat works on the amount of light transmission control.

It is expected that nanotechnology will occupy the first place in the IT industry around the world, in the coming years and affect life significantly. These modern technologies do an interest in the Internet field, where it will develop business in engineering, medicine, weapons and a lot of important things in the life of human beings.

\section{POTENTIAL DANGERS OF NANOTECHNOLOGY}

Despite the broad applications of nanotechnology, there are considerable concerns about the possibility of side effects on the use of this technique in human life [3].

The nanoparticles can be easily implemented through the skin, lungs and intestinal organs without knowing the impact on human health. On the other hand, the inhalation of nanomaterial's (such as nanoparticles, balls nanoparticles, carbon nanoparticles, pipes ...) lead to the entry of these materials into the body, and then it reaches to the brain [3].

There is no regulation or clear laws which determine the damage and dangers resulting from the use of nanomaterials with a high volume of the same type of particles. Some researchers have claimed that Nanoparticles may cause the death or rodent damage of the brain in a fish, where other studies have indicated air pollution when increasing the concentration of nanoparticles in the air, which may lead to an increase in disease and death.

In general, it is extremely recommended for workers in nanotechnology to take all kinds of extreme caution necessary to avoid inhalation of nanomaterials of all types, or when contact with human skin [3].

\section{CONCLUSION}

This paper has discussed the nanotechnology. While it seems the definition of Nan science is easy, the definition of nanotechnology is more difficult, due to the hyperlinked and interference of various applied fields. This paper has defined nanotechnology based on some research and introduced different types of nanotechnology in details. In addition, this paper has discussed the uses of these types in the recent years and presented some of the possible side effects that can be caused by using nanotechnology.

\section{REFERENCES}

[1] R. I. Bahar, D. Hammerstrom, J. Harlow, W. H. Joyner, Jr., C. Lau, D. Marculescu, A. Orailoglu, and M. Pedram, "Architectures for Silicon Nanoelectronics and Beyond," Computer, Vol. 40, No 1, pp. 25-33, 2007.

[2] W. Seifert, M. Borgstrom, K. Deppert, K. A. Dick, J. Johansson, M. W. Larsson, T. Martensson, N. Skold, C. P. T. Svensson, B. A. Wacaser, L. R. Wallenberg, and L. Samuelson, J. Cryst. Growth 272, 2004, 211.

[3] Saud Alarifi, Daoud Ali, Saad Alkahtani, Ankit Verma, Maqusood Ahamed, Mukhtar Ahmed and Hisham A Alhadlaq,. "Induction of oxidative stress, DNA damage, and apoptosis in a malignant human skin melanoma cell line after exposure to zinc oxide nanoparticles," International Journal of Nanomedicine (Dovepress Journal), Volume 2013:8 Pages 983 - 993 March 2013."

[4] Mostafa Ghasemi, Manal Ismail, Siti Kartom Kamarudin, Kasra Saeedfar, Wan Ramli Wan Daud, Sedky H.A. Hassan, Lee Yook Heng, Javed Alam and Sang-Eun Oh, "Carbon nanotube as an alternative cathode support and catalyst for microbial fuel cells", Elsevier, Applied Energy, Volume 102, , Pages 1050-1056 February 2013"

[5] "NanoSi (2015) Dr. Munir Nayfeh, President/Chief Technical Officer, Available at NanoSi:: Home. 2015. NanoSi:: Home19th March 2015."

[6] Ritu Rani,, Gagan Kumar, Khalid Mujasam Batoo and M. Singh "Electric and Dielectric Study of Zinc Substituted Cobalt Nanoferrites Prepared by Solution Combustion Method," American Journal of Nanomaterials, 1, pp 9-12, 2013."

[7] Shams Tabrez Khan, Maqusood Ahamed, Abdulaziz Al-Khedhairy and Javed Musarrat,"Biocidal effect of copper and zinc oxide nanoparticles on human oral microbiome and biofilm formation," Materials Letters (Elsevier), Volume 97, 15 Pages 67-70, April 2013 
[8] A. DeHon and M. J. Wilson, "Nanowire-based sublithographic programmable logic arrays," The 2004 ACM/SIGDA 12th international symposium on Field programmable gate arrays, 2004."

[9] Maqusood Ahamed, Hisham A. Alhadlaq, Javed Alam, M. A. Majeed Khan, Daoud Ali and Saud Alarafi "Iron Oxide Nanoparticle-induced Oxidative Stress and Genotoxicity in Human Skin Epithelial and Lung Epithelial Cell Lines," Current Pharmaceutical Design, Volume 19, ISSUE 37, Pages 6681-6690

[10] Maqusood Ahamed, Hisham A. Alhadlaq, M. A. Majeed Khan, Mohd. "Selective killing of cancer cells by iron oxide nanoparticles mediated through reactive oxygen species via p53 pathway", Javed Akhtar, Journal of Nanoparticle Research (Springer), December 2012

[11] Jianping Yang, Dengke Shen, Lei Zhou, Wei Li, Xiaomin Li, Chi Yao, Rui Wang, Ahmed Mohamed El-Toni, Fan Zhang and Dongyuan Zhao,Spatially Confined Fabrication of Core-Shell Gold Silica for Near-Infrared Controlled Photothermal Drug Release", American Chemical Society, Chemistry of Materials, 25 (15), pp: 3030-3037 16 July 2013

[12] Khaled Mahmoud and Mohammed Zourob, " $\mathrm{Fe}_{3} \mathrm{O}_{4} / \mathrm{Au}$ nanoparticles/lignin modified microspheres as effectual surface enhanced Raman scattering (SERS) substrates for highly selective and sensitive detection of 2,4,6trinitrotoluene (TNT)", Analyst, 2013,138, 2712-2719, Impact Factor: 3.969

[13] Sungho Kim, Bongsik Choi, Meehyun Lim, Jinsu Yoon, Juhee Lee, Hee-Dong Kim, and Sung-jun Choi, "Pattern Recognition Using Carbon Nanotube Synaptic Transistors with an Adjustable Weight Update Protocol," ACS Publications, 2017.

[14] Mahesh S, Karthick Raja A, Ezhil Kumar M and Harish V, A Review on Explosive Detection Methods for Security Enhancement of Home security system. International Journal of Electrical Transformation and Restructuring, Vol.1, Issue 1, March 2016, p 35-41.

[15] Guoxing Li, Jinhua Sun, Wenpeng Hou, Shidong Jiang, Yong Huang and Jianxin Geng, "Three-Dimensional Porous Carbon Composites Containing High Sulfur Nanoparticle Content for High-Performance Lithium-Sulfur Batteries," Nature Communication, 2016, DOI: 10:1038/ncomms 10601.

[16] Liuqi Zhang, Xiaolei Yang, Qi Jiang, Pengyang Wang, Zhigang Yin, Xingwang Zhang, Hairen Tan, Yong (Michael) Yang, Mingyang Wei, Brandon R. Sutherland, Edward H. Sargent and Jingbi You, "Untra-Bright and Highly Efficient Inorganic Based Perovskite Light-Emitting Diodes", Nature Communication, 2017, DOI: $10.1038 /$ ncomms 15640 . 